\title{
The CLAS Excited Baryon Program at JLab
}

\author{
Volker Crede \\ Florida State University, Tallahassee, FL 32306, USA
}

\begin{abstract}
Nucleons are complex systems of confined quarks and exhibit characteristic spectra of excited states. Highly excited nucleon states are sensitive to details of quark confinement which is poorly understood within Quantum Chromodynamics (QCD), the fundamental theory of strong interactions. Thus, measurements of excited states and the corresponding determination of their properties are needed to come to a better understanding of how confinement works in nucleons. However, the excited states of the nucleon cannot simply be inferred from cleanly separated spectral lines. Quite the contrary, a spectral analysis in nucleon resonance physics is challenging because of the fact that the resonances are broadly overlapping states which decay into a multitude of final states involving mesons and baryons. To provide a consistent and complete picture of an individual nucleon resonance, the various possible production and decay channels must be treated in a multichannel framework that permits separating resonance from background contributions. Very often, resonances reveal themselves more clearly through interference with dominant amplitudes. These interference terms can be isolated via polarization observables. The current CLAS effort is to utilize highly-polarized hydrogen and deuterium targets as well as polarized photon beams toward a complete measurement of a large number of reaction channels.
\end{abstract}

Keywords: Transition Form Factors, Hadron Spectroscopy, Photoproduction, Polarization PACS: $13.40 . \mathrm{Gp}, 13.60 . \mathrm{Le}, 13.60 . \mathrm{Rj}, 25.20 . \mathrm{Lj}, 29.27 . \mathrm{Hj}$

\section{INTRODUCTION}

The CLAS excited baryon program has two main components. One is to establish the systematics of the spectrum, which provides information on the nature of the effective degrees of freedom in strong QCD. At Jefferson Laboratory (JLab), extensive data sets on photo- as well as electro-production of pseudo-scalar mesons $(\pi, 2 \pi, \eta$, and $\mathrm{K})$ and vector mesons $(\rho, \omega, \phi)$ have been accumulated using the CEBAF Large Acceptance Spectrometer (CLAS). The next generation of CLAS experiments will use polarized targets to investigate double-polarization observables using both hydrogen and deuterium. The other component is to probe the internal structure of excited baryon states and provide information about confinement by measuring the transition form factors from the nucleon ground state to excited baryon states.

\section{RESONANCE TRANSITION FORM FACTORS}

Electron beams are ideal to measure the resonance transition form factors from the nucleon ground state to excited baryon states. These measurements probe the internal structure of the excited states and provide information about the confining forces of the 3-quark system. The $\Delta(1232)$ resonance is the lowest and most prominent baryon excitation, and the $N \rightarrow \Delta$ transition has served as a prototype for testing theoretical

CP947, VII Latin American Symposium on Nuclear Physics and Applications edited by R. Alarcon, P. L. Cole, C. Djalali, and F. Umeres

(C) 2007 American Institute of Physics 978-0-7354-0461-8/07/\$23.00 

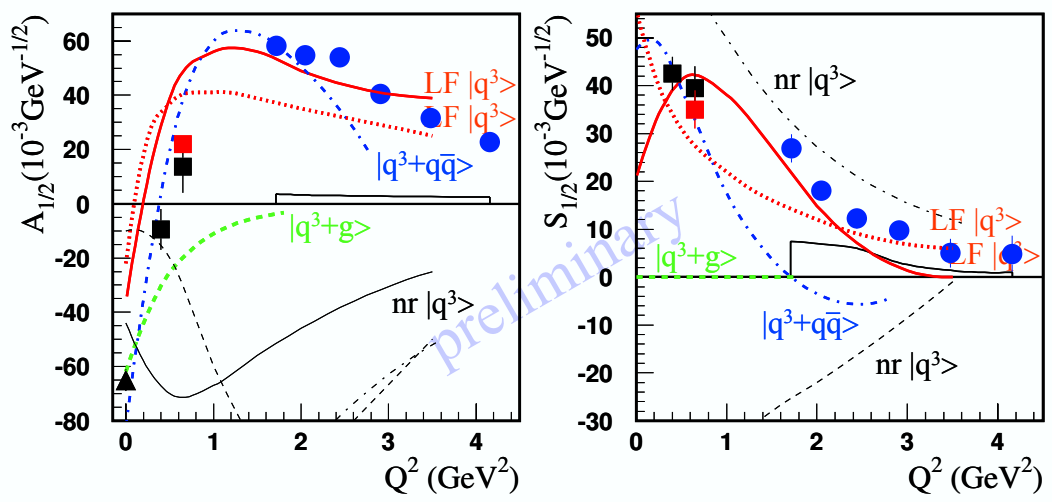

FIGURE 1. The extracted $\gamma N \rightarrow N^{*}(1440)$ form factors compared with results from different models.

models of baryon structure. The $Q^{2}$ dependence of the electromagnetic multipoles in the $N \rightarrow \Delta$ transition is sensitive to the evolution from soft non-perturbative physics to hard processes and perturbative QCD. A recent Jefferson Lab experiment has extended the measurement of the electromagnetic $N \rightarrow \Delta$ transition to $Q^{2}=6 \mathrm{GeV}^{2} / c^{2}$, the highest momentum transfer yet achieved [1]. It is found that the magnetic form factor decreases with $Q^{2}$ more steeply than the proton (elastic) magnetic form factor and that the multipole ratio $E_{1+} / M_{1+}$ is small and negative, indicating strong helicity nonconservation.

Moreover, accurate data of the transition form factors for several other higher mass $\mathrm{N}^{*}$ states have been extracted in recent years. Figure 1 shows the transition form factor for the $\mathrm{N}(1440) \mathrm{P}_{11}$ (Roper) resonance and illustrates the sensitivity of these measurements to the baryon structure. A comparison with relativistic quark model calculations shows that the state has a quark substructure at small distances eliminating models of this state describing it as a hybrid baryon or a meson-baryon molecule.

\section{BARYON SPECTROSCOPY}

The mass spectrum of hadron resonances is clearly organized according to flavor content, spin and parity. For intermediate and long-distance phenomena such as hadron properties, the full complexity of QCD emerges, including nonlinearity and confinement, and is a strong obstacle to understanding hadronic phenomena at a fundamental level. Lattice-QCD studies are making progress towards the solution of the QCD Lagrangian in the low-energy regime and for bound states, but more development is required. In the mean time, quark models have been developed to predict the properties of hadronic states. Thus, the primary goals of hadron physics are to determine the relevant degrees of freedom at different scales, to relate them to each other, and ultimately to the parameters and fundamental fields of QCD. 
A long-standing question in hadron physics is whether the large number of so-called missing baryon resonances really exists, i.e. experimentally not established baryon states which are predicted by all quark models based on three constituent quark effective degrees of freedom. Nearly all existing data on non-strange production of baryon resonances result from $\mathrm{N} \pi$ scattering experiments. However, quark models predict strong couplings of these missing states to $\gamma \mathrm{N}$ as well as single-meson final states like $\mathrm{N} \eta, \mathrm{N} \eta^{\prime}$, $\mathrm{N} \rho, \mathrm{N} \omega$, but also, especially at higher energies, to complex two-meson final states such as $\mathrm{N} \pi \pi$ or $\mathrm{N} \pi \eta$. At center-of-mass energies below $1.7 \mathrm{GeV}$, the single-pion production channel dominates both the pion and photoabsorption cross sections. As the c.m. energy increases towards $2.0 \mathrm{GeV}$ and beyond, two- and even three-meson final states become dominant, and it is in this important energy region that the masses and partial widths of baryon resonances are poorly determined. The current challenge is to identify some of the higher-lying baryon states with masses between 1.7 and $2.2 \mathrm{GeV} / c^{2}$.

\section{Hyperon Production}

Photoproduction of strangeness off the proton leading to $\mathrm{K} \Lambda$ and $\mathrm{K} \Sigma$ states is a fundamental process that is part of the broader field of elementary pseudoscalar-meson production. Much of the recent experimental effort has been motivated by theoretical calculations suggesting that strangeness photoproduction might be a fertile place to search for non-strange baryon resonances. For the first time, differential cross sections for the reaction $\gamma \mathrm{p} \rightarrow \mathrm{K}^{* 0} \Sigma^{+}$have been determined over a broad range of angles [2]. A comparison with a theoretical model based on the vector and tensor $\mathrm{K}^{*}$-quark couplings (after adjusting the model's two parameters in a fit to the data) shows good general agreement with the data (Fig. 2).
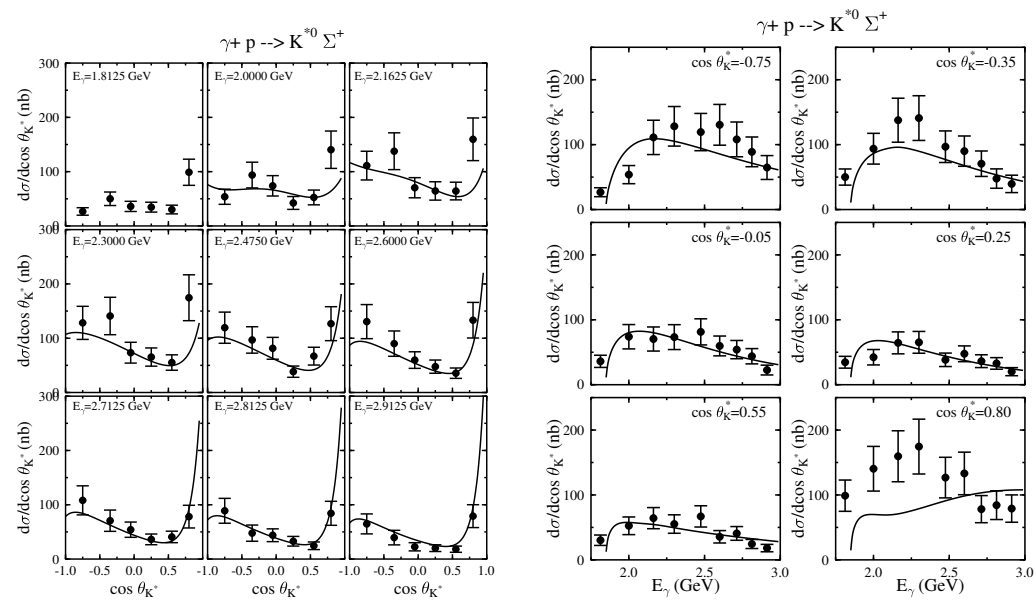

FIGURE 2. Differential cross sections for the reaction $\gamma \mathrm{p} \rightarrow \mathrm{K}^{* 0} \Sigma^{+}$as a function of angle $\theta_{\mathrm{K}^{*}}$ (left) and of energy (right) [2]. The curves are from [3] using vector and tensor couplings adjusted to give the best fit to the data. Error bars include systematic uncertainties. 
Differential cross sections for $\mathrm{K}^{+} \mathrm{Y}$ for $\Lambda$ and $\Sigma^{0}$ hyperons have been published recently by the CLAS collaboration [4]. Together with the results published by other groups, these cross sections have been tests for a number of single-channel theoretical models. As these models were adjusted to the new cross section measurements, there was a claim for a specific new baryonic resonance around $1.9 \mathrm{GeV} / c^{2}$ decaying to $\mathrm{K}^{+} \Lambda$. However, no unique solution of the baryon resonance content is currently available. Moreover, no model provides an overall good representation of the differential and single polarization observable data and thus, measurements of new observables are needed in order to achieve better understanding from $\mathrm{K}^{+} \Lambda$ and $\mathrm{K}^{+} \Sigma^{0}$.

Since $\Lambda$ polarization can be measured by using its self-analyzing decay distribution, it is possible to extract double and triple polarization observables and measure spin transfer from the photon to the hyperon. A surprising result is that $\Lambda$ 's appear to be $100 \%$ polarized if produced by circularly-polarized photons, independent of the photon energy and scattering angle [5] (see later section for more details).

\section{Double-Pion Electroproduction}

Many candidates for new baryon states have been found in various channels for further confirmation in experiments to be performed with polarized beams and targets. For example, the very accurate two-pion electroproduction data prove to be very effective in the search for baryon states decaying primarily into the resonant $\pi \Delta$ or $\rho \mathrm{N}$ final states. Measurements of the two-pion decay channels complement the single-pion results, and are especially important for the higher-mass states above $1.7 \mathrm{GeV} / c^{2}$.
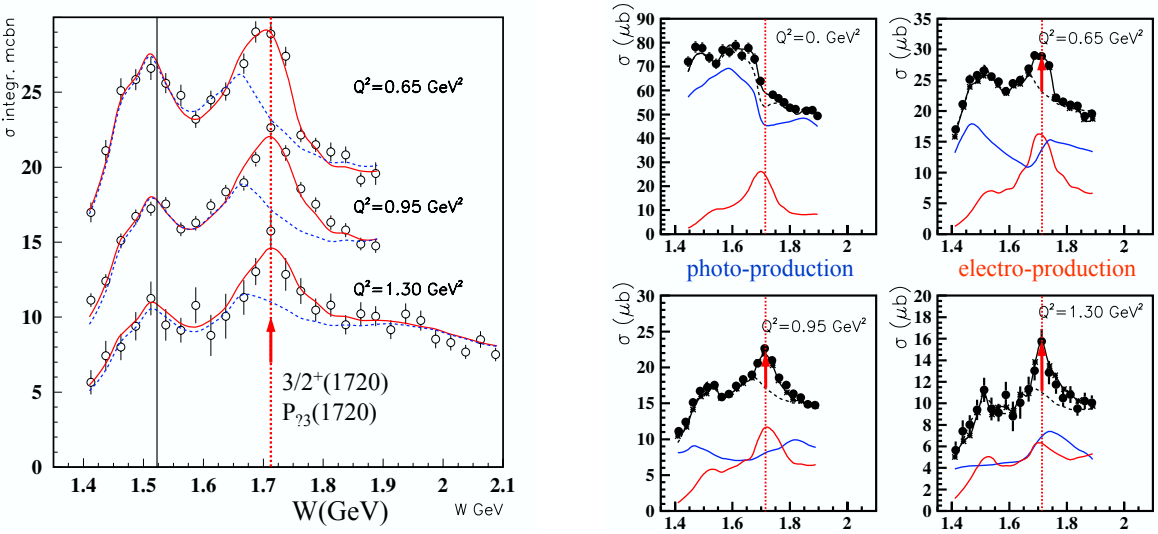

FIGURE 3. Integrated cross sections for the reaction $\gamma p \rightarrow \pi^{+} \pi^{-} p$ as function of the invariant hadronic mass. The (red) solid curves are from fits using the JM06 model [6]. The (blue) dashed curves are obtained when the contributions of a $J^{P}=\frac{3}{2}^{+}$candidate at $1.72 \mathrm{GeV} / c^{2}$ are turned off. 
The JLab-MSU collaboration has developed an isobar model (JM06) with treediagram mechanisms and additional phenomenological terms to analyze the very extensive $\pi^{+} \pi^{-}$photo- and electro-production data from JLab [6]. The authors claim that all significant $2 \pi$ contributions have been identified in the analysis. Preliminary data on the $Q^{2}$ evolution of the electro-couplings of the $\Delta(1620) \mathrm{S}_{31}, \mathrm{~N}(1720) \mathrm{P}_{13}, \mathrm{~N}(1700) \mathrm{D}_{13}$, and $\Delta(1700) \mathrm{D}_{33}$ states have become available for the first time. Figure 3 illustrates some results of the JM06 $\pi^{+} \pi^{-}$model at different $Q^{2}$. The analysis shows evidence for a $J^{P}=\frac{3}{2}^{+}$state at $1720 \mathrm{MeV} / c^{2}$ which decays strongly into $\pi \Delta$. This state could be different from the conventional $\mathrm{N}(1720) \mathrm{P}_{13}$ state decaying predominantly into $\rho \mathrm{N}$ as listed by the Particle Data Group in 2006 [7].

\section{THE CLAS POLARIZATION PROGRAM}

The goal of the current CLAS polarization program is to use highly-polarized hydrogen and deuterium targets as well as polarized photon beams to completely measure a large number of reaction channels. These data, together with the already accumulated unpolarized cross sections, will form the basis for a much more efficient and much less ambiguous search for new baryon states than has been possible to date. In a coupledchannel analysis, these data will allow extraction of $\mathrm{N}^{*}$ resonance parameters even of the well known states with much improved accuracy.

The photon beam facilities in Hall-B at JLab provide energy-tagged unpolarized and polarized bremsstrahlung photons with energies up to $5.5 \mathrm{GeV}$, providing access to

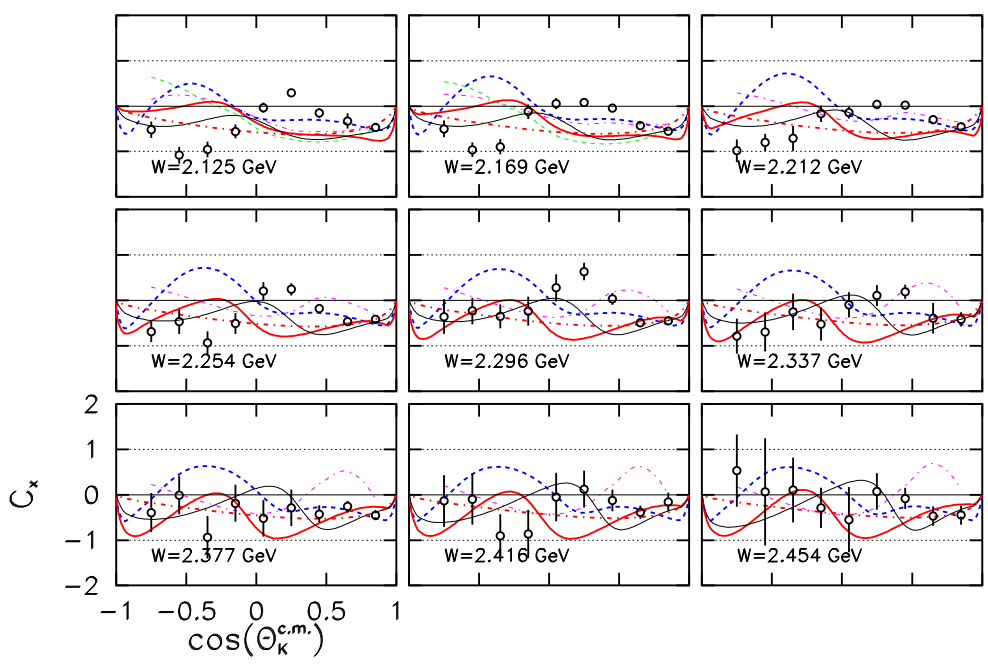

FIGURE 4. The observable $C_{x}$ for the reaction $\vec{\gamma}+p \rightarrow K^{+}+\vec{\Lambda}$, plotted as a function of the kaon angle. The panels are for increasing values of $W$ in steps of $50 \mathrm{MeV}$. The open circles represent the CLAS results, the curves are from different models [5]. 


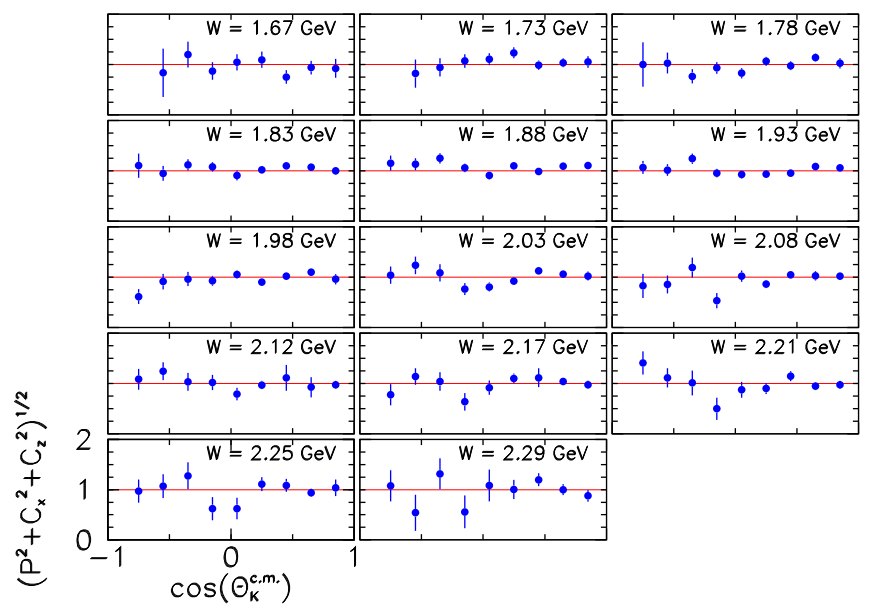

FIGURE 5. The magnitude of the $\Lambda$ hyperon polarization observable vector $R_{\Lambda}=\sqrt{P^{2}+C_{x}^{2}+C_{z}^{2}}$ in the same binning as Fig. 4. $R_{\Lambda}$ is consistent with unity over all values of $W$ and kaon angle.

hadronic masses up to $3 \mathrm{GeV} / \mathrm{c}^{2}$. The first CLAS experiments using unpolarized photon beams and unpolarized hydrogen and deuterium targets have measured the differential cross sections of various pseudo-scalar meson production channels. Subsequent experiments have used a linearly-polarized photon beam to measure the beam asymmetries $\Sigma$ [8]. Recently, CLAS has accumulated data for reactions off the neutron using an unpolarized deuterium target and circularly- as well as linearly-polarized photons [9].

\section{Beam-Recoil Observables $C_{x}$ and $C_{z}$ in Hyperon Photoproduction}

When the photon beam is unpolarized, parity conservation in electromagnetic production allows induced polarization $P$ of the hyperon only along the axis perpendicular to the reaction plane $\hat{\gamma} \times \hat{K}$. However, when the incoming photons are circularly polarized, that is, when the photons are spin polarized parallel or anti-parallel to the beam direction, giving them net helicity, then this polarization may be transferred in whole or in part to the spin orientation of the produced hyperons within the reaction plane. $C_{x}$ and $C_{z}$ characterize the polarization transfer from a circularly-polarized incident photon beam to a recoiling hyperon along orthogonal axes in the reaction plane. CLAS has measured these observables for center-of-mass energies $W$ between 1.6 and $2.53 \mathrm{GeV}$, and for $-0.85<\cos \theta_{\mathrm{K}^{+}}^{\text {c.m. }}<+0.95$. As an example, Figure 4 shows the observable $C_{x}$ for the reaction $\vec{\gamma}+p \rightarrow K^{+}+\vec{\Lambda}$, plotted as a function of the kaon angle. For the $\Lambda$, the polarization transfer coefficient along the photon momentum axis, $C_{z}$, was found to be near unity for a wide range of energy and kaon production angles. The associated 
transverse polarization coefficient, $C_{x}$, is smaller than $C_{z}$ by a roughly constant difference of unity (Fig. 4). Most significantly, the total $\Lambda$ polarization vector, including the induced polarization $P$, has magnitude consistent with unity at all measured energies and production angles when the beam is fully polarized (Fig. 5). This is kinematically not required and the origin is esentially unknown. All existing hadrodynamic models are in poor agreement with these results.

\section{The Double-Polarization Program}

The next generation of CLAS photo-production experiments will use polarized targets to study double-polarization observables more generally on both hydrogen and deuterium. The polarized Frozen-Spin Target (FROST) is in the final testing phase at JLab and will be used to measure all beam-target double polarization observables for a large number of final states. These experiments are scheduled to begin in 2007 and many of the accessible observables will be measured for the first time.

The FROST program at JLab anticipates to take data for all four combinations of beam and target polarization, thus providing (almost) complete sets of measurements for $\pi^{0}, \pi^{+}, \eta, \eta^{\prime}$, and $\pi^{+} \pi^{-}$photoproduction. The additional determination of the recoil polarization in hyperon production, completes polarization measurements for the reactions $\gamma \mathrm{p} \rightarrow \mathrm{K} \Sigma$ and $\gamma \mathrm{p} \rightarrow \mathrm{K} \Lambda$ [10]. The program was awarded beam time to determine all observables over a large range in production angle and energy with anticipated statistical errors between \pm 0.05 and \pm 0.07 .

To study the isospin decomposition of the resonance amplitude, the measurements off polarized protons will be complemented by those off polarized neutrons provided by the HD polarized target. The latter was built at Brookhaven National Laboratory and is currently planned to be transferred to JLab for data taking in 2009/2010.

\section{The FROzen-Spin Target (FROST)}

The frozen-spin (butanol) target will be positioned in the geometrical center of CLAS with a minimum amount of material in the path of outgoing particles. The target cryostat is of horizontal type with a pipe of about $200 \mathrm{~cm}$ in length and $25 \mathrm{~cm}$ in diameter. Since CLAS is a magnetic spectrometer, its operational characteristics are very sensitive to the additional magnetic field produced by the target. For this reason, the target material will be dynamically polarized by microwave irradiation in a strong magnetic field of $5 \mathrm{~T}$ at a temperature of about $1 \mathrm{~K}$ outside of CLAS. After maximum polarization is reached, the cryostat will be switched to holding (or frozen-spin) mode using a much lower magnetic field of $0.5 \mathrm{~T}$ at a temperature of $\approx 50 \mathrm{mK}$ and moved back into CLAS. For a butanol target, the proton polarization of initially about $90 \%$ generally decreases slowly with a relaxation time of typically several days under the conditions outlined above. This is sufficiently long for a useful polarized-target experiment [11].

The target cell is $50 \mathrm{~mm}$ long and $15 \mathrm{~mm}$ in diameter. The length is a compromise between conflicting demands for count rate and cooling requirements. Butanol has a 
dilution factor (fraction of polarizable nucleons) of $10 / 74 \approx 13.5 \%$ and an effective density of $0.611 \mathrm{~g} / \mathrm{cm}^{3}$. An average degree of polarization for both longitudinal and transverse polarization of about $80 \%$ is anticipated.

For the cylindrical target volume in question, approximately $20 \mathrm{~mW}$ of microwave power will be necessary for the polarization process at $0.5 \mathrm{~K}$ considering a polarization build-up time of about 30 minutes. Under experimental run conditions the heat load from the photon beam will be about $1 \mu \mathrm{W}$. This implies that in frozen-spin mode at $0.5 \mathrm{~T}$ and $50 \mathrm{mK}$, the refrigerator should provide a cooling power of a few $\mu \mathrm{W}$. The only technique that satisfies these conditions is a ${ }^{3} \mathrm{He} /{ }^{4} \mathrm{He}$ dilution refrigerator.

Polarization Observables in $\pi^{+} \pi^{-}$Production. In the past, the $\mathrm{p} \pi^{+} \pi^{-}$final state has been treated often as arising from either of the quasi two-body states $\Delta \pi$ or $N \rho$, followed by the decay of the $\Delta$ or the $\rho$. This Isobar Approach has been reasonably successful. However, it is considered important that the polarization observables for such processes be measured and elucidated in a more general framework, one that goes beyond the quasi two-body assumption. The formalism and terminology for the various polarization observables of interest in double-meson photoproduction can be found in [12].

For the reaction $\gamma \mathrm{p} \rightarrow \mathrm{p} \pi \pi$, all polarization observables are 5-fold differential and more important, there are 15 observables in double-meson production as opposed to only 7 observables in single-meson production. For a circularly-polarized beam and an unpolarized target, the helicity difference has been measured by the CLAS Collaboration [13], providing the only double-pion observable to date.

\section{REFERENCES}

1. M. Ungaro et al. [CLAS Collaboration], Phys. Rev. Lett. 97, 112003 (2006).

2. I. Hleiqawi et al. [CLAS Collaboration], Phys. Rev. C 75 (2007) 042201.

3. Q. Zhao, J.S. Al-Khalili and C. Bennhold, Phys. Rev. C 64, 052201 (2001).

4. R. Bradford et al. [CLAS Collaboration], Phys. Rev. C 73, 035202 (2006).

5. R. Bradford et al. [CLAS Collaboration], Phys. Rev. C 75, 035205 (2007).

6. V.I. Mokeev, V.D. Burkert, et. al., Prepared for International Workshop on the Physics of Excited Baryons (NSTAR 05), Tallahassee, Florida, 10-15 Oct 2005, edited by S. Capstick, V. Crede, and P. Eugenio, p. 47-56

7. W.M. Yao et al. [Particle Data Group], J. Phys. G 33, 1 (2006).

8. P. Cole and J. Salamanca, contributions to these Proceedings.

9. E. Munevar, contribution to these Proceedings.

10. F.J. Klein et al., Search for Missing Nucleon Resonances in the Photoproduction of Hyperons using Polarized Photon Beams and Polarized Targets, JLab Proposal E02-112.

D.I. Sober et al., Helicity Structure of Pion Photoproduction, JLab Proposal E03-102.

S. Strauch et al., Pion Production From a Polarized Target, JLab Proposal E03-105.

E. Pasyuk et al., Polarization Observables in $\eta$ Photoproduction with CLAS, JLab Proposal E05-012.

V. Crede et al., Measurement of $\pi^{+} \pi^{-}$Photoproduction in Double-Polarization Experiments using CLAS, JLab Proposal E06-013.

11. C. Hanretty, contribution to these Proceedings.

12. W. Roberts and T. Oed, Phys. Rev. C71, 055201 (2005).

13. S. Strauch et al. [CLAS Collaboration], Phys. Rev. Lett. 95 (2005) 162003. 